\title{
Comparison of formol-acetone concentration method with that of the direct iodine preparation and formol-ether concentration methods for examination of stool parasites
}

\author{
Feleke Moges ${ }^{1,2}$, Yeshambel Belyhun ${ }^{1}$, Moges Tiruneh ${ }^{1}$, Yenew Kebede ${ }^{3}$, Andargachew Mulu ${ }^{1,4}$, Afework Kassu ${ }^{1,5}$, \\ Kahsay Huruy ${ }^{6}$
}

\section{Abstract}

Background: Formol-ether concentration technique is taken as a gold standard method to detect most intestinal parasites; however, because of its low safety and hazardous impact a need for better technique has a paramount importance.

Objective: To evaluate a formol- acetone concentration method in comparison with the conventional direct iodine preparation and formol- ether concentration methods in detecting intestinal parasites.

Methods: A total of 382 stool samples were collected from Tseda elementary school children, in 2006. Samples were processed and examined using formol-acetone concentration, the direct iodine stained smear, and formol-ether concentration methods.

Results: Formol-ether detected $79.1 \%$ of parasites followed by formol-acetone $(73.6 \%)$ and direct iodine preparation $(50.3 \%)$. Statistical $(\mathrm{P}<0.05)$ difference was observed for the detection of over all positivity of any parasites between the two concentration methods. However, the sensitivity, specificity, and positive predicative value of formol-acetone were $88.1 \%, 81.3 \%$, and $94.7 \%$, respectively respective to formol-ether method. Almost similar detection ability was also observed by the two concentration methods for A. lumbricoids, H. nana, T. trichuira, and S. stercoralis. However, there was difference in the detection rate of hookworm and $S$. mansoni.

Conclusions: for safety and hazard free laboratory set up, this new method might be used as an alternative choice for formol-ether concentration method. [Ethiop. J. Health Dev. 2010;24(2):148-151]

\section{Introduction}

The most commonly used methods for detecting intestinal parasites from stool examination include, direct and concentration techniques $(1,2)$. Studies $(2,3)$ indicated that of all conventional concentration techniques, the formol- ether concentration technique, which consists of ether as a fat solvent for detecting out parasites from intestinal debris and thus increases the positivity rates, is believed to be superior over the other methods. However, this technique has been considered to be still disadvantageous since the use of ether may be hazardous for health to laboratory personnel. Ether is explosive, contains anaesthetic vapours, has potential toxicity such as respiratory irritation, and causes cardiovascular depression and narcosis $(3,4)$. Moreover, it can be a possible cause of mutagen, if inhaled, or absorbed through the skin often with harmful long term health effects like neurotoxicity or cancer (4). For this technique, finding a comparable substitute has been actively sought and continued and various studies have indicated that solvents like ethyl acetate, tween, acetone and petrol can be a better substitute $(5,6,7)$. However, among these solvents, acetone has been rated as "safe" in comparison to the ether effect on health (8) and had an equivalent detection rate of parasites to ether (5).

Therefore, in accordance with this assumption, in areas where there is high prevalence of parasites and a need of replacement of ether by a hazard free solvent as well as effective diagnostic technique has a paramount importance. Thus, this study aimed at evaluating a formol-acetone concentration method in comparison with the conventional direct iodine preparation and formolether concentration methods.

\begin{abstract}
Methods
Study design and specimen collection: A crosssectional study conducted in Tseda elementary school located $23 \mathrm{~km}$ south east of Gondar town, Northwest Ethiopia, from December-March, 2006. The students were stratified by age and sex which was sampled by using systematic random sampling technique using the
\end{abstract}

\footnotetext{
${ }^{1}$ Department of Microbiology and Parasitology, College of Medicine and Health Sciences, University of Gondar, P. O. Box 196, E-mail: yeshi4s@yahoo.com, Tele. 0912029138, Fax-251-058-114 79 Gondar, Ethiopia;

${ }^{2}$ Pharmaceutical Microbiology/Biotechnology Division, College of Pharmaceutical Sciences, Andhra University, Visakhapatnam-530003, Andhra Pradesh, India;

${ }^{3}$ Branch Chief for Laboratory, Center for Disease Control and Prevention (CDC)-Ethiopia, US Embassy, P. O. Box 1014;

${ }^{4}$ Institute of Virology, Medical Faculty, University of Leipzig, Germany;

${ }^{5}$ Division of Allergy and Clinical Immunology, Department of Medicine, University of Colorado Denver, USA;

${ }^{6}$ Department of Medical Laboratory Technology, College of Medicine and Health Sciences, University of Gondar, P.

O. Box 196, Gondar, Ethiopia
} 
master list of the schools. Considering a $95 \%$ confidence level, high background prevalence of intestinal parasites in the area (40\%) and 5\% margin of error, a total of 386 study subjects were included in the study.

Fresh stool was collected from each study subject in a clean, wide mouthed and water proof container. The samples were protected from contamination with water, soil and urine. A portion of the specimen was processed immediately at the site for direct iodine preparation examination using diluted iodine following standard procedure (9). Another portion of the same sample was sent to Gondar University Hospital laboratory for further processing using concentration technique. For concentration techniques; about $1 \mathrm{gm}$ of the stool sample of each subject was taken in a $15 \mathrm{ml}$ centrifuge tube containing $10 \mathrm{ml}$ of $10 \%$ formalin. Formol-acetone concentration technique was processed and prepared for microscopy with exactly the same procedure to formolether concentration technique (9).

To avoid inter observer variation; two technicians were involved blindly in the procedure; one was involved in the preparation of slides for direct iodine preparation, formol-ether and formol-acetone concentration techniques, while the other did microscopy. The sensitivity and specificity of the direct microscopy and formol-acetone concentration methods were assessed by considering formol- ether concentration as gold standard.

Ethical considerations: Approval for this study was granted by the Ethics Committee of the Research and Publication office of University of Gondar. The data was collected after a written informed consent was sought from the school officials and each study participants and infected children were communicated and has got antiparasitic treatment.

\section{Results}

A total of 382 stool samples were collected and examined from $171(44.8 \%)$ male and $211(55.2 \%)$ female children and high detection rate was observed by formol-ether 302 (79.1) and by formol acetone $281 \quad(73.6 \%),(\mathrm{P}<0.05)$ followed by direct iodine preparation $192(50.3 \%$ ) (Table 1). Qualitatively, all parasites (10 species) which were detected by the routine methods (formol-ether and direct iodine stained) were also detected by the new method but with different rates of recovery (Table 1).

Table 1: Detection of stool parasites with direct iodine preparation, formol ether and formol acetone concentration methods

\begin{tabular}{|c|c|c|c|c|}
\hline ( & $\begin{array}{l}\text { Direct iodine } \\
\text { N (\%) }\end{array}$ & $\begin{array}{l}\text { Formol ether } \\
\mathbf{N}(\%)\end{array}$ & $\begin{array}{l}\text { Formol acetone } \\
\mathrm{N}(\%)\end{array}$ & p-value ${ }^{\star}$ \\
\hline \multicolumn{5}{|c|}{ A. lumbricoides } \\
\hline Positive & $116(30.4)$ & 202 (52.9) & $196(51.3)$ & \multirow[t]{2}{*}{0.41} \\
\hline Negative & 266 ( 69.6) & $180(47.1)$ & $186(48.7)$ & \\
\hline \multicolumn{5}{|l|}{ Hookworm } \\
\hline Positive & $15(3.9)$ & $75(19.6)$ & $36(9.4)$ & \multirow[t]{2}{*}{$<0.001$} \\
\hline Negative & 367 (96.1) & $307(80.4)$ & $346(90.6)$ & \\
\hline \multicolumn{5}{|l|}{ S. mansoni } \\
\hline Positive & $13(3.4)$ & 108 ( 28.3) & $53(13.9)$ & \multirow[t]{2}{*}{$<0.001$} \\
\hline Negative & $369(96.6)$ & $274(71.7)^{\prime}$ & $329(86.1)$ & \\
\hline \multicolumn{5}{|c|}{ E. vermicularis } \\
\hline Positive & $2(0.5)$ & $4(1.0)$ & $4(1.0)$ & \multirow[t]{2}{*}{$\mathrm{Nd}$} \\
\hline Negative & 308 (99.5) & $378(99.0)$ & $378(99.0)$ & \\
\hline \multicolumn{5}{|l|}{ T.trichiura } \\
\hline Positive & $6(1.6)$ & $20(5.2)$ & $15(3.9)$ & \multirow[t]{2}{*}{0.27} \\
\hline Negative & $376(98.4)$ & $362(94.8)$ & $367(96.1)$ & \\
\hline \multicolumn{5}{|c|}{ Giardia cyst } \\
\hline Positive & $48(12.6)$ & $59(15.4)$ & $68(17.8)$ & \multirow[t]{2}{*}{0.14} \\
\hline Negative & $334(87.4)$ & $323(84.6)$ & $314(82.2)$ & \\
\hline \multicolumn{5}{|l|}{ H. nana } \\
\hline Positive & $25(6.5)$ & $37(9.7)$ & $33(8.6)$ & \multirow[t]{2}{*}{0.39} \\
\hline Negative & $357(93.5)$ & $345(90.3)$ & $349(91.4)$ & \\
\hline \multicolumn{5}{|c|}{ S. Stercoralis } \\
\hline Positive & $15(3.9)$ & $24(6.3)$ & $16(4.2)$ & \multirow[t]{2}{*}{0.10} \\
\hline Negative & $367(96.1)$ & $358(93.7)$ & $366(95.8)$ & \\
\hline \multicolumn{5}{|c|}{ Taenia species } \\
\hline Positive & $1(0.3)$ & $0(0)$ & $1(0.3)$ & \multirow[t]{2}{*}{$\mathrm{Nd}$} \\
\hline Negative & $381(99.7)$ & $382(100.0)$ & $381(99.7)$ & \\
\hline \multicolumn{5}{|c|}{ Ameoba cyst } \\
\hline Positive & $0(0)$ & $1(0.3)$ & $2(0.5)$ & \multirow[t]{2}{*}{$\mathrm{Nd}$} \\
\hline Negative & $382(100.0)$ & 381 (99.7) & $380(99.5)$ & \\
\hline Total & $192(50.3)$ & $302(79.1)$ & $281(73.6)$ & 0.005 \\
\hline
\end{tabular}


Detection of $A$. lumbricoides was $202(52.9 \%)$ by formolether, $196(51.3 \%)$ by formol acetone $(\mathrm{P}=0.41)$, and 116 (30.4\%) by direct iodine methods. T. trichuira detection rate was found to be almost similar by formol-ether, 20 $(5.2 \%)$ and formol-acetone, 15 (3.9\%), $(\mathrm{P}=0.27)$. $H$. nana was also detected in almost similar rates by formol ether, $36(9.4 \%)$ and formol-acetone $33(8.6 \%),(\mathrm{P}=0.39)$. S. stercoralis detection rate was $24(6.3 \%)$ and $16(4.2 \%)$, $(\mathrm{P}=0.10)$ by formol-ether and formol-acetone methods, respectively. Giardia cysts were found to be higher in formol-acetone method $68(17.8 \%)$ followed by formolether $59(15.4 \%),(\mathrm{P}=0.14)$ and direct iodine preparation method 48 (12.6\%), however, no statistical difference was observed (Table 1).

Hookworm species was found to be higher by formolether 75 (19.6\%) followed by formol-acetone $36(9.4 \%)$, $(\mathrm{P}<0.001)$ and direct iodine preparation 15 (3.9\%). $S$. mansoni was observed more by formol-ether method 108 $(28.3 \%)$ than formol-acetone method, 53 (13.9\%), $(\mathrm{P}<$ $0.001)$ and very low detection by direct iodine preparation method $13(3.9 \%)$.

Methods compared in this study showed records for double, triple and quadruple parasite infections of $32.5 \%$, $9.7 \%$, and $2.4 \%$, respectively for formol-ether concentration, $20.9 \%, 6.0 \%$, and $1.6 \%$ for formolacetone concentration, and $9.2 \%, 1.3 \%, 0.3 \%$ for direct iodine preparation. Regarding multiple infections; double, triple and quadruple infection rates were recorded more in formol-ether method while detection of single infection was more in formol-acetone method.

The sensitivity of formol acetone was found to be $88.1 \%$ and that of direct iodine preparation was $60.9 \%$. However, specificity of formol-acetone was $81.3 \%$ where as that of direct iodine preparation was $90.0 \%$. The positive and negative predictive values were $94.7 \%$ and $64.4 \%$, respectively for formol-acetone method. Direct iodine preparation had also $95.8 \%$ and $37.9 \%$ of positive and negative predictive values, respectively.

\section{Discussion}

In this study, a wide range of parasites was detected by the new formol- acetone method. In comparison to parasite recovery, our results confirmed that formolether concentration method $(79.1 \%)$ is quite higher and effective than the direct iodine preparation method $(50.3 \%) \quad(\mathrm{P}<0.001)$, but almost comparable to the new formol- acetone concentration method (73.6\%) although there was no statistical significance $(\mathrm{P}>0.05)$. This result agrees favorably with others similar studies $(3,4,5)$. As reported by Parija et al (5) intestinal parasites recovery rate of $65.26 \%$ for formol-ether and $34.74 \%$ for direct smear methods, which clearly indicated the superiority of the formol-ether technique over direct iodine preparation method. However, the current study tried to see the specificity, sensitivity, positive and negative predictive values of the two concentration methods. Other similar studies failed to report the comparison between the two concentration methods using this approaches and it was very important before decision has made to use acetone instead of ether.

In the detection of A. lumbricoides, T. trichuira, E. vermicularis, and $H$. nana there was no statistical difference between the two concentration methods and this was in line with other similar study (10).

In $S$. stercoralis detection, the two concentration methods showed no difference and almost similar detection rate was observed between direct iodine preparation and formol-acetone concentration which is not in line with the suggestion that a negative result does not necessarily indicate the unequivocal absence of the infection unless more than one stool examination technique is used for diagnosis of S. stercoralis (11).

Direct iodine preparation missed $22.5 \%, 3.9 \%, 0.5 \%$ and $2.9 \%$ of infected individuals with $A$. lumbricoides, $T$. trichuira, E. vermicularis, and $H$. nana, respectively as compared to formol-ether method. As compared to formol-ether method, formol acetone method missed $1.6 \%$ of $A$. lumbricoids and T. trichuira. However, the reverse was true for Giardia cyst which was $2.4 \%$ higher rate of detection by using formol acetone method than formol ether method. Though direct iodine preparation is quick to prepare and inexpensive it can miss parasites (ova, cysts and larvae). Thus in this study, a significant number of the infected population was missed by direct iodine preparation method as compared to the new method.

The detection of hookworm and S. mansoni was found to be higher using formol-ether concentration method than formol-acetone and direct iodine preparation method. Direct iodine preparation was 3 times less for the detection of the hookworms and 8 times less for detection of S. mansoni. This might support the idea that KetoKatez method is generally recommended for the diagnosis of $S$. mansoni infection $(10,12)$.

The pattern of sensitivity and specificity also suggest that formol-acetone concentration method could suffice for routine examination of stool specimens for intestinal parasites, particularly for studies and /or setups that prefer formol-ether concentration techniques. In this regard, we failed to compare with similar studies and that's why we were interested to see these parameters to test the effectiveness of the new method. However, the superiority of formol-ether over the iodine wet mount was reported elsewhere (5) which was also in line with the current study.

Multiple infections, mostly with 2, 3, and 4 parasites in the same individual, were most common in formol- ether concentration than formol- acetone concentration and 
direct iodine preparation methods. However, for detection of single parasite formol- acetone concentration $(45.1 \%)$ has been superior to formol- ether concentration (34.6\%) and direct iodine preparation method (39.5\%). In this regard, double, triple and quadruple parasite infections were better detected in the two concentration methods than direct iodine preparation method. This may be because; ether and acetone can be helpful for dissolving debris and fats from stool samples so as to increase the detection of formol- ether and formolacetone concentration techniques, respectively. No comparison was made to other similar studies and the current study was the only one to try to see such additional evaluation approaches of the methods used.

In conclusion, we found that the formol- acetone method had a higher detection power of parasites than direct iodine method. With the exception of hookworm and S.mansoni, formol-acetone method was comparable to the formol- ether concentration method. Therefore, for safety and hazard free laboratory set up, we recommend that the formol-acetone concentration technique as an alternative method.

\section{Acknowledgements}

We thank the Research and Publication Office of University of Gondar for funding the project. Authors would like to thank Belay Anagaw, Martha Alemayehu and Fikre Aserie for their technical assistance. We also thank the study participants and Tseda Elementary School principals.

\section{References}

1. Faust EC, D'Antoni JS, Odom V, Odom V, Miller MJ, Peres C, Sawitz W, et al. A critical study of clinical laboratory technicians for the diagnosis of protozoan cycts and helminth eggs in feces. Am J Trop Med 1938;18:169-183.

2. Ritchie LS. An ether sedimentation technique for routine stool examinations. Bull US Army Med Dept 1948;8:326.
3. Young KH, Bullock SL, Melvin DM, Spruill, CL. Ethyl acetate as a substitute for diethyl ether in the Formalin-ether sedimentation technique. J Clin Microbiol 1979;10:852-53.

4. Erdman D. Clinical Comparison of Ethyl Acetate and Diethyl Ether in the Formalin-Ether Sedimentation Technique. Journal of Clinical Microbiology 1981;14(5):483-485.

5. Parija SC, Bhattacharya S, Padhan P, Shivaprakash MR. Evaluation of formalin-acetone sedimentation in the concentration of stool for intestinal parasites. Journal of Tropical Doctor 2003;33:163-164.

6. Truant AL, Elliott SH, Kelly MT, Smith JH. Comparison of formalin-ethyl ether sedimentation and zinc sulfate floatation techniques for the detection of intestinal parasites. Clin Microbiol 1981;5:882-884.

7. Pakdad K, Ahmadi NA. Tween as a Substitute for Diethyl Ether in the Formalin-Ether Sedimentation Technique. Iranian J Publ Health 2007;36(4):91-95.

8. Boswell MV, Collins, VJ. Diethyl ether and chloroform. In: Collins VJ, eds. Physiologic and pharmacologic bases of anesthesia. Pennsylvania, Williams \& Wilkins, 1996;650-62.

9. Cheesbrough M. District laboratory practice in tropical countries, $3^{\text {rd }}$ ed. Part One, Cambridge University Press, United Kingdom, 2000;214-215.

10. Knight WB, Hiatt RA, Cline BL, Ritchie LS. A modification of the formol- ether concentration technique for increased sensitivity in detecting Schistosoma mansoni eggs. Am J Trop Med Hyg 1976;25(6):818-823.

11. Indris MA, Al-Jabri, AM. Usefulness of Keto-Katez and trichome staining as diagnostic methods for parasitic infections in clinical laboratories. SQU J Sci Resp 2001;3(2):65-68.

12. Kongs A, Marks G, Verle PA, Van der Stuyft P. The unreliability of the Kato-Katz technique limits its usefulness for evaluating $S$. mansoni infections. Tropical Medicine and International Health 2001;6(3):163-169. 